\title{
Possible involvement of a single common mechanism in the cardioprotective effects of pre-/per-/postconditioning
}

Takashi Koyama*

Keywords: Ischemia reperfusion injury, Postconditioning with lactate-enriched blood, Revascularization, ST-segment elevation myocardial infarction

\section{To the Editor}

Clinical translation of pre-/per-/postconditioning has long been attempted, with no substantial success so far. The precise mechanisms underlying the cardioprotective effects of these approaches remain to be elucidated. Knowledge of the precise mechanisms may help determine when and whom to apply these approaches and how to achieve maximal cardioprotective effects.

My colleagues and I recently reported a modified ischemic postconditioning protocol, postconditioning with lactate-enriched blood (PCLeB) (Koyama et al. 2013,2014). PCLeB comprises intermittent reperfusion and timely coronary injections of lactated Ringer's solution (Fig. 1), aimed at increasing the delay in recovery from intracellular acidosis by impeding lactate washout. Through this modification, we specifically targeted reperfusion-induced hypercontracture, which develops within minutes of reperfusion of the ischemic myocardium. The strong mechanical force generated by hypercontracture disrupts the cell skeleton, leading to irreversible cell injury. The higher tissue lactate concentrations achieved by PCLeB during reperfusion may impede interactions between myofilaments and attenuate hypercontracture development. Despite the small-scale nature of the study, good long-term outcomes associated with reduced plasma NT-proBNP levels have been reported in patients

*Correspondence: koyamas@me.com

Department of Cardiology, Saitama Municipal Hospital, 2460 Mimuro, Midori-ku, Saitama City, Saitama 336-8522, Japan with ST-segment elevation myocardial infarction treated using PCLeB (Koyama et al. 2020).

Remote ischemic perconditioning (McLeod et al. 2017) appears to create a condition similar to that created by PCLeB in reperfused ischemic myocardium. Intermittent limb ischemia releases tissue lactate, which is locally produced and accumulated during limb ischemia, into the systemic circulation. Thus, remote ischemic perconditioning can be regarded as a maneuver that enables reperfusion of the ischemic myocardium with "lactateenriched blood."

Instead of attenuating force generation, force dispersion may be another approach. Pacing postconditioning is a procedure in which ventricular pacing is implemented during reperfusion (Babiker 2016). Pacinginduced dyssynchrony may disperse the force generated by hypercontracture because the timing of the peak systole differs between cells, and the force generated by myocardial contraction cannot be fully integrated, which may weaken the shear force imposed on each myocardial cell.

Regarding ischemic preconditioning, I previously reported the possibility that its cardioprotective effects may reside in the delayed onset of reperfusion injury (Koyama 2021). In other words, a longer duration of prolonged ischemia may be needed for reperfusion injury to occur in preconditioned hearts. If this is true, a large difference in infarct size observed between the control and preconditioned hearts is determined by whether reperfusion injury has occurred. Preconditioning has been demonstrated to delay the increase in intracellular $\mathrm{Ca}^{2+}$ 


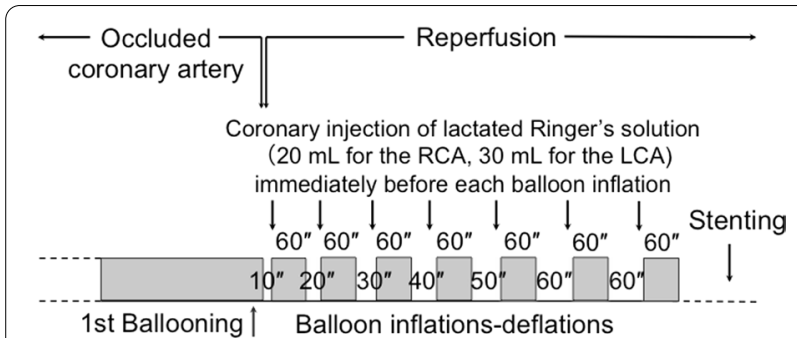

Fig. 1 Overview of the protocol for postconditioning with lactate-enriched blood. The duration of each brief reperfusion is prolonged stepwise from 10 to $60 \mathrm{~s}$. At the end of each brief reperfusion, lactate is supplied by injecting lactated Ringer's solution into the culprit coronary artery. The balloon is rapidly inflated at the lesion site to trap the lactate inside the ischemic myocardium. Each ischemic period lasts for $60 \mathrm{~s}$. After seven cycles of balloon inflation and deflation, full reperfusion is performed, followed by stenting. LCA, left coronary artery; RCA, right coronary artery. (Reprinted from Koyama et al. 2014)

concentrations $\left(\left[\mathrm{Ca}^{2+}\right] \mathrm{i}\right)$ during subsequent prolonged ischemia (Dekker et al. 1996). Therefore, prolonged ischemia may take longer to achieve the threshold level of $\left[\mathrm{Ca}^{2+}\right]$ i for developing hypercontracture after reperfusion in the preconditioned hearts because hypercontracture develops with elevated $\left[\mathrm{Ca}^{2+}\right] \mathrm{i}$ and re-energization of myofilaments by adenosine triphosphate production after reperfusion. When prolonged ischemia is terminated relatively early, myocardial injury caused by reperfusion-induced hypercontracture would occur in control hearts but not in preconditioned hearts; thus, a large difference in the infarct size was observed between the two (Murry et al. 1986).

In conclusion, the cardioprotective effects of pre-/per-/ postconditioning may be explained using a single common key concept, reperfusion-induced hypercontracture.

\section{Abbreviations \\ $\left[\mathrm{Ca}^{2+}\right]$ i: Intracellular $\mathrm{Ca}^{2+}$ concentrations; PCLeB: Postconditioning with lactate-enriched blood.}

\section{Acknowledgements}

I would like to thank Editage [http://www.editage.com] for editing and reviewing this manuscript for English language.

\section{Authors' contributions}

Not applicable because this is a single-author paper.
Funding

None.

Availability of data and materials

Not applicable.

\section{Declarations}

Ethics approval and consent to participate

Not applicable.

\section{Consent for publication}

Not applicable.

\section{Competing interests}

The author declares no competing interest relevant to this work.

Received: 25 May 2021 Accepted: 14 July 2021

Published online: 19 July 2021

\section{References}

Babiker FA (2016) Pacing postconditioning: recent insights of mechanism of action and probable future clinical application. Med Princ Pract 25(Suppl 1):22-28. https://doi.org/10.1159/000381916

Dekker LR, Fiolet JW, VanBavel E, Coronel R, Opthof T, Spaan JA et al (1996) Intracellular $\mathrm{Ca} 2+$, intercellular electrical coupling, and mechanical activty in ischemic rabbit papillary muscle. Effects of preconditioning and metabolic blockade. Circ Res 79(2):237-246. https://doi.org/10.1161/01. res.79.2.237

Koyama T (2021) An alternative viewpoint for the cardioprotective effects of ischemic preconditioning. J Xiangya Med 6:10. https://doi.org/10.21037/ jxym-20-107

Koyama T, Shibata M, Moritani K (2013) Ischemic postconditioning with lactate-enriched blood in patients with acute myocardial infarction. Cardiology 125:92-93. https://doi.org/10.1159/000350595

Koyama T, Niikura H, Shibata M, Moritani K, Shimada M, Baba A et al (2014) Impact of ischemic postconditioning with lactate-enriched blood on early inflammation after myocardial infarction. IJC Metab Endocr 2:30-34. https://doi.org/10.1016/j.ijcme.2014.02.002

Koyama T, Munakata M, Akima T, Kanki H (2020) Reduced plasma NT-proBNP levels months after myocardial infarction postconditioned with lactateenriched blood. Cardiology 145(4):199-202. https://doi.org/10.1159/ 000505903

McLeod SL, lansavichene A, Cheskes S (2017) Remote ischemic perconditioning to reduce reperfusion injury during acute ST-segment-elevation myocardial infarction: a systematic review and meta-analysis. J Am Heart Assoc 6(5):e005522. https://doi.org/10.1161/JAHA.117.005522

Murry CE, Jennings RB, Reimer KA (1986) Preconditioning with ischemia: a delay of lethal cell injury in ischemic myocardium. Circulation 74(5):11241136. https://doi.org/10.1161/01.CIR.74.5.1124

\section{Publisher's Note}

Springer Nature remains neutral with regard to jurisdictional claims in published maps and institutional affiliations. 